\title{
Toda Tau Functions with Quantum Torus Symmetries
}

\author{
K. Takasaki
}

\begin{abstract}
The quantum torus algebra plays an important role in a special class of solutions of the Toda hierarchy. Typical examples are the solutions related to the melting crystal model of topological strings and 5D SUSY gauge theories. The quantum torus algebra is realized by a $2 \mathrm{D}$ complex free fermion system that underlies the Toda hierarchy, and exhibits mysterious "shift symmetries". This article is based on collaboration with Toshio Nakatsu.
\end{abstract}

Keywords: Toda hierarchy, melting crystal model, quantum torus algebra.

\section{Introduction}

This paper is a review of our recent work $[1,2]$ on an integrable structure of the melting crystal model of topological strings [3] and 5D gauge theories [4]. It is shown here that the partition function of this model, on being suitably deformed by special external potentials, is essentially a tau function of the Toda hierarchy [5]. A technical clue to this observation is a kind of symmetries (referred to as "shift symmetries") in the underlying quantum torus algebra. These symmetries enable us, firstly, to convert the deformed partition function to the tau function and, secondly, to show the existence of hidden symmetries of the tau function. These results can be extended to some other Toda tau functions that are related to the topological vertex [6] and the double Hurwitz numbers of the Riemann sphere [7].

\section{Quantum torus algebra}

Throughout this paper, $q$ denotes a constant with $|q|<1$, and $\Lambda$ and $\Delta$ denote the $\mathbf{Z} \times \mathbf{Z}$ matrices

$$
\Lambda=\sum_{i \in \mathbf{Z}} E_{i, i+1}=\left(\delta_{i+1, j}\right), \quad \Delta=\sum_{i \in \mathbf{Z}} i E_{i i}=\left(i \delta_{i j}\right) .
$$

Their combinations

$$
v_{m}^{(k)}=q^{-k m / 2} \Lambda^{m} q^{k \Delta} \quad(k, m \in \mathbf{Z})
$$

satisfy the commutation relations

$$
\left[v_{m}^{(k)}, v_{n}^{(l)}\right]=\left(q^{(l m-k n) / 2}-q^{(k n-l m) / 2}\right) v_{m+n}^{(k+l)}
$$

of the quantum torus algebra. This Lie algebra can thus be embedded into the Lie algebra $\operatorname{gl}(\infty)$ of $\mathbf{Z} \times \mathbf{Z}$ matrices $A=\left(a_{i j}\right)$ for which $\exists N$ such that $a_{i j}=0$ for $|i-j|>N$.

To formulate a fermionic realization of this Lie algebra, we introduce the creation/annihilation operators $\psi_{i}, \psi_{i}^{*}(i \in \mathbf{Z})$ with anti-commutation relations

$$
\psi_{i} \psi_{j}^{*}+\psi_{j}^{*} \psi_{i}=\delta_{i+j, 0}
$$

$$
\begin{aligned}
\psi_{i} \psi_{j}+\psi_{j} \psi_{i} & =0 \\
\psi_{i}^{*} \psi_{j}^{*}+\psi_{j}^{*} \psi_{i}^{*} & =0
\end{aligned}
$$

and the $2 \mathrm{D}$ free fermion fields

$$
\psi(z)=\sum_{i \in \mathbf{Z}} \psi_{i} z^{-i-1}, \quad \psi^{*}(z)=\sum_{i \in \mathbf{Z}} \psi_{i}^{*} z^{-i} .
$$

The vacuum states $\langle 0|| 0$,$\rangle of the Fock space and its$ dual space are characterized by the vacuum conditions

$$
\begin{array}{ll}
\psi_{i}|0\rangle=0(i \geq 0), & \psi_{i}^{*}|0\rangle=0(i \geq 1), \\
\langle 0| \psi_{i}=0(i \leq-1), & \langle 0| \psi_{i}^{*}=0(i \leq 0) .
\end{array}
$$

To any element $A=\left(a_{i j}\right)$ of $\mathrm{gl}(\infty)$, one can associate the fermion bilinear

$$
\begin{gathered}
\widehat{A}=\sum_{i, j \in \mathbf{Z}} a_{i j}: \psi_{-i} \psi_{j}^{*}:, \\
: \psi_{-i} \psi_{j}^{*}:=\psi_{-i} \psi_{j}^{*}-\left\langle 0\left|\psi_{-i} \psi_{j}^{*}\right| 0\right\rangle .
\end{gathered}
$$

These fermion bilinears form a one-dimensional central extension $\mathrm{gl}(\infty)$ of $\mathrm{gl}(\infty)$. The special fermion bilinears $[1,2]$

$$
\begin{aligned}
V_{m}^{(k)}= & \widehat{v_{m}^{(k)}}= \\
& q^{k / 2} \oint \frac{\mathrm{d} z}{2 \pi i} z^{m}: \psi\left(q^{k / 2} z\right) \psi^{*}\left(q^{-k / 2} z\right):
\end{aligned}
$$

satisfy the commutation relations

$$
\begin{aligned}
{\left[V_{m}^{(k)}, V_{n}^{(l)}\right]=} & \left(q^{(l m-k n) / 2}-q^{(k n-l m) / 2}\right) . \\
& \left(V_{m+n}^{(k+l)}-\frac{q^{k+l}}{1-q^{k+l}} \delta_{m+n, 0}\right)
\end{aligned}
$$

for $k$ and $l$ with $k+l \neq 0$ and

$$
\begin{aligned}
{\left[V_{m}^{(k)}, V_{n}^{(-k)}\right]=} & \left(q^{-k(m+n) / 2}-q^{k(m+n) / 2}\right) . \\
& V_{m+n}^{(0)}+m \delta_{m+n, 0} .
\end{aligned}
$$


Thus $\mathrm{gl}(\infty)$ contains a central extension of the quantum torus algebra, in which the $\widehat{u(1)}$ algebra is realized by

$$
J_{m}=V_{m}^{(0)}=\widehat{\Lambda^{m}} \quad(m \in \mathbf{Z})
$$

\section{$3 \quad$ Shift symmetries}

Let us introduce the operators

$$
\begin{aligned}
& G_{ \pm}=\exp \left(\sum_{k=1}^{\infty} \frac{q^{k / 2}}{k\left(1-q^{k}\right)} J_{ \pm k}\right) \\
& W_{0}=\sum_{n \in \mathbf{Z}} n^{2}: \psi_{-n} \psi_{n}^{*}:
\end{aligned}
$$

$G_{ \pm}$'s play the role of "transfer matrices" in the melting crystal model $[3,4]$. $W_{0}$ is a fermionic form of the so called "cut-and-join" operator for Hurwitz numbers [8].

$G_{ \pm}$and $q^{W_{0} / 2}$ induce the following two types of "shift symmetries" $[1,2]$ in the (centrally extended) quantum torus algebra.

- First shift symmetry

$$
\begin{aligned}
& G_{-} G_{+}\left(V_{m}^{(k)}-\delta_{m, 0} \frac{q^{k}}{1-q^{k}}\right)\left(G_{-} G_{+}\right)^{-1}= \\
& (-1)^{k}\left(V_{m+k}^{(k)}-\delta_{m+k, 0} \frac{q^{k}}{1-q^{k}}\right)
\end{aligned}
$$

- Second shift symmetry

$$
q^{W_{0} / 2} V_{m}^{(k)} q^{-W_{0} / 2}=V_{m}^{(k-m)}
$$

\section{Toda tau function in melting crystal model}

A general tau function of the 2D Toda hierarchy [5] is given by

$$
\begin{aligned}
& \tau(s, \boldsymbol{T}, \overline{\boldsymbol{T}})= \\
& \left\langle s\left|\exp \left(\sum_{k=1}^{\infty} T_{k} J_{k}\right) g \exp \left(-\sum_{k=1}^{\infty} \bar{T}_{k} J_{-k}\right)\right| s\right\rangle,
\end{aligned}
$$

where $\boldsymbol{T}=\left(T_{1}, T_{2}, \cdots\right)$ and $\overline{\boldsymbol{T}}=\left(\bar{T}_{1}, \bar{T}_{2}, \cdots\right)$ are time variables of the Toda hierarchy, $\langle s|$ and $|s\rangle$ are the ground states

$$
\left\langle s\left|=\left\langle-\infty\left|\cdots \psi_{s-1}^{*} \psi_{s}^{*}, \quad\right| s\right\rangle=\psi_{-s} \psi_{-s+1} \cdots\right|-\infty\right\rangle
$$

in the charge- $s$ sector of the Fock space, and $g$ is an element of $\mathrm{GL}(\infty)=\exp (\operatorname{gl}(\infty))$.

On the other hand, the partition function $Z(Q, s, \boldsymbol{t})$ of the deformed melting crystal model $[1,2]$ can be cast into the apparently similar (but essentially different) form

$$
Z(s, \boldsymbol{t})=\left\langle s\left|G_{+} e^{H(\boldsymbol{t})} Q^{L_{0}} G_{-}\right| s\right\rangle,
$$

where $Q$ and $\boldsymbol{t}=\left(t_{1}, t_{2}, \cdots\right)$ are coupling constants of the model, and $H(\boldsymbol{t})$ and $L_{0}$ the following operators:

$$
\begin{aligned}
H(\boldsymbol{t}) & =\sum_{k=1}^{\infty} t_{k} H_{k}, \quad H_{k}=V_{0}^{(k)}, \\
L_{0} & =\sum_{n \in \mathbf{Z}} n: \psi_{-n} \psi_{n}^{*}: .
\end{aligned}
$$

The shift symmetries (8) and (9) imply the operator identity

$$
\begin{aligned}
G_{+} e^{H(\boldsymbol{t})} G_{+}^{-1}= & \exp \left(\sum_{k=1}^{\infty} \frac{t_{k} q^{k}}{1-q^{k}}\right) G_{-}^{-1} q^{-W_{0} / 2} . \\
& \exp \left(\sum_{k=1}^{\infty}(-1)^{k} t_{k} J_{k}\right) q^{W_{0} / 2} G_{-} .
\end{aligned}
$$

Inserting this identity and using the fact that

$$
\begin{aligned}
& \langle s| G_{-}^{-1} q^{-W_{0} / 2}=q^{-s(s+1)(2 s+1) / 12}\langle s|, \\
& q^{-W_{0} / 2} G_{+}^{-1}|s\rangle=q^{-s(s+1)(2 s+1) / 12}|s\rangle,
\end{aligned}
$$

we can rewrite $Z(s, \boldsymbol{t})$ as

$$
\begin{aligned}
Z(Q, s, \boldsymbol{t})= & \exp \left(\sum_{k=1}^{\infty} \frac{t_{k} q^{k}}{1-q^{k}}\right) \\
& q^{-s(s+1)(2 s+1) / 6} \tau(s, \boldsymbol{T}, \mathbf{0}), \\
T_{k}= & (-1)^{k} t_{k}
\end{aligned}
$$

where the $\mathrm{GL}(\infty)$ element $g$ defining the tau function is given by

$$
g=q^{W_{0} / 2} G_{-} G_{+} Q^{L_{0}} G_{-} G_{+} q^{W_{0} / 2} .
$$

Actually, the shift symmetries imply the operator identity

$$
\begin{aligned}
G_{-}^{-1} e^{H(\boldsymbol{t})} G_{-}= & \exp \left(\sum_{k=1}^{\infty} \frac{t_{k} q^{k}}{1-q^{k}}\right) G_{+} q^{W_{0} / 2} \\
& \exp \left(\sum_{k=1}^{\infty}(-1)^{k} t_{k} J_{-k}\right) q^{-W_{0} / 2} G_{+}^{-1}
\end{aligned}
$$

as well. This leads to another expression of $Z(Q, s, \boldsymbol{t}$,$) in which \tau(s, \boldsymbol{T}, \mathbf{0})$ is replaced with $\tau(s, \mathbf{0},-\boldsymbol{T})$.

The existence of different expressions can be explained by the intertwining relations

$$
J_{k} g=g J_{-k} \quad(k=1,2, \ldots),
$$

which, too, are a consequence of the shift symmetries. These intertwining relations imply the constraints

$$
\left(\partial_{T_{k}}+\partial_{\bar{T}_{k}}\right) \tau(s, \boldsymbol{T}, \overline{\boldsymbol{T}})=0 \quad(k=1,2, \ldots)
$$


on the tau function. The tau function $\tau(s, \boldsymbol{T}, \overline{\boldsymbol{T}})$ thereby becomes a function $\tau(s, \boldsymbol{T}-\overline{\boldsymbol{T}})$ of the difference $\boldsymbol{T}-\overline{\boldsymbol{T}}$. In particular, $\tau(s, \boldsymbol{T}, \mathbf{0})$ and $\tau(s, \mathbf{0},-\boldsymbol{T})$ coincide. The reduced function $\tau(\boldsymbol{T}, s)$ may be thought of as a tau function of the 1D Toda hierarchy.

(15) are a special case of the more general intertwining relations

$$
\begin{aligned}
& \left(V_{m}^{(k)}-\delta_{m, 0} \frac{q^{k}}{1-q^{k}}\right) g= \\
& Q^{-k} g\left(V_{-2 k-m}^{(-k)}-\delta_{2 k+m, 0} \frac{q^{-k}}{1-q^{-k}}\right) .
\end{aligned}
$$

We can translate these relations to the language of the Lax formalism of the Toda hierarchy. A study on this issue is now in progress.

\section{Other models}

The following Toda tau functions can be treated more or less in the same way as the foregoing tau function. We shall discuss this issue elsewhere.

1. The generating function of the two-leg amplitude $W_{\lambda \mu}$ in the topological vertex [6] is a Toda tau function determined by

$$
g=q^{W_{0} / 2} G_{+} G_{-} q^{W_{0} / 2} .
$$

2. The generating function of double Hurwitz numbers of the Riemann sphere [7] is a Toda tau function determined by

$$
g=e^{-\beta W_{0}} Q^{L_{0}} .
$$

The parameter $q$ is interpreted as $q=e^{-\beta}$.

\section{Acknowledgement}

This work has been partly supported by the JSPS Grants-in-Aid for Scientific Research No. 19104002, No. 21540218 and No. 22540186 from the Japan Society for the Promotion of Science.

\section{References}

[1] Nakatsu, T., Takasaki, K.: Melting crystal, quantum torus and Toda hierarchy, Comm. Math. Phys. 285 (2009), 445-468.

[2] Nakatsu, T., Takasaki, K.: Integrable structure of melting crystal model with external potential, Advanced Studies in Pure. Math. vol. 59 (Math. Soc. Japan, 2010), pp. 201-223.

[3] Okounkov, A., Reshetikhin, N., Vafa, C.: Quantum Calabi-Yau and classical crystals, In: P. Etingof, V. Retakh, I. M. Singer (eds.), The unity of mathematics, Progr. Math. 244, Birkhäuser, 2006, pp. 597-618.

[4] Maeda, T., Nakatsu, T., Takasaki, K., Tamakoshi, T.: Five-dimensional supersymmetric YangMills theories and random plane partitions, JHEP 0503 (2005), 056.

[5] Takasaki, K., Takebe, T.: Integrable hierarchies and dispersionless limit, Rev. Math. Phys. 7 (1995), 743-808.

[6] Zhou, J.: Hodge integrals and integrable hierarchies, arXiv:math.AG/0310408.

[7] Okounkov, A.: Toda equations for Hurwitz numbers, Math. Res. Lett. 7 (2000), 447-453.

[8] Kazarian, M.: KP hierarchy for Hodge integrals, Adv. Math. 221 (2009), 1-21.

Kanehisa Takasaki

E-mail: takasaki@math.h.kyoto-u.ac.jp

Graduate School of Human

and Environmental Studies

Kyoto University

Yoshida, Sakyo, Kyoto, 606-8501, Japan 